\title{
11.1
}

\section{Микрополосковые фильтры с широкими полосами пропускания}

\author{
() Б.А. Беляев ${ }^{1,2}$, С.А. Ходенков ${ }^{1}$, И.В. Говорун ${ }^{1,3}$, А.М. Сержантов ${ }^{1,2}$ \\ ${ }^{1}$ Сибирский государственный университет науки и технологий им. акад. М.Ф. Решетнева, Красноярск, Россия \\ ${ }^{2}$ Сибирский федеральный университет, Красноярск, Россия \\ ${ }^{3}$ Институт фризики им. Л.В. Киренского СО РАН, Красноярск, Россия \\ E-mail: belyaev@iph.krasn.ru
}

Поступило в Редакцию 13 октября 2020г.

В окончательной редакции 15 декабря 2020 г.

Принято к публикации 24 декабря 2020 г.

\begin{abstract}
Разработаны новые микрополосковые конструкции полосно-пропускающих фильтров на основе фильтра нижних частот, в котором часть или все отрезки высокоомных микрополосковых линий соединены с экраном шлейфами. Фильтры обладают высокими частотно-селективными свойствами, а их относительная ширина полосы пропускания попадает в интервал 30-150\%. Экспериментальный образец фильтра с центральной частотой полосы пропускания $2 \mathrm{GHz}$ и ее относительной шириной $70 \%$, изготовленный на подложке из поликора толщиной $1 \mathrm{~mm}$, имеет площадь подложки $46 \times 21 \mathrm{~mm}$. Показано хорошее согласие измеренных амплитудно-частотных характеристик фильтра с характеристиками, рассчитанными с помощью численного электродинамического анализа его 3D-модели.
\end{abstract}

Ключевые слова: полосно-пропускающий фильтр, микрополосковый резонатор, диэлектрическая подложка, амплитудно-частотная характеристика.

DOI: 10.21883/PJTF.2021.07.50796.18581

Увеличение скорости передачи информации является одной из важнейших задач в цифровых системах радиосвязи, при решении которой нередко прибегают к расширению рабочей полосы частот. Для таких систем требуются сверхширокополосные полосно-пропускающие фильтры, обладающие высокой избирательностью, конструкции которых активно разрабатываются в последние годы. Такие фильтры, как известно, легко реализуются каскадным соединением фильтра нижних частот (ФНЧ) с фильтром верхних частот $[1,2]$. Однако большой интерес представляют более простые и технологичные в изготовлении микрополосковые конструкции фильтров на связанных резонаторах, в которых частично удалена металлизация в экране для увеличения взаимодействия между резонаторами $[3,4]$ или использованы трехмодовые резонаторы [5]. При этом для фильтров с относительной шириной полосы пропускания $\Delta f / f_{0}$ больше $100 \%$ лучше подходят микрополосковые конструкции на нерегулярных четвертьволновых резонаторах, связанных либо кондуктивно [6,7], либо индуктивно [8].

Нами исследована новая конструкция сверхширокополосного полосно-пропускающего фильтра на основе микрополоскового ФНЧ седьмого порядка, топология проводников и амплитудно-частотные характеристики (АЧХ) которого представлены на рис. 1. Волновое сопротивление портов на входе и выходе фильтра равно $50 \Omega$. Подложка этого ФНЧ толщиной $1 \mathrm{~mm}$ из поликора с относительной диэлектрической проницаемостью $\varepsilon=9.8$ при частоте среза фильтра $2.5 \mathrm{GHz}$ имеет сравнительно небольшую площадь $(40 \times 16 \mathrm{~mm})$ благодаря свернутому вдвое нерегулярному проводнику. На представленном рисунке обозначены размеры регу- лярных отрезков полоскового проводника конструкции в миллиметрах. Сворачивание проводника ФНЧ не только уменьшает длину подложки, но и увеличивает крутизну склона АЧХ [9] из-за появления дополнительной электромагнитной связи между несоседними резонаторами [10].

ФНЧ становится полосно-пропускающим фильтром, если с помощью шлейфов соединить с экраном часть или все узкие отрезки его нерегулярного проводника. В этом случае каждый шлейф не только играет роль параллельной индуктивности, как в фильтрах верхних частот, но и является взаимной индуктивностью соседних резонаторов, величина которой определяет их взаимодействие. На рис. 2 представлены АЧХ четырех фильтров, в первом из которых замкнуты на экран только два отрезка, обозначенные римской цифрой I на топологии проводников ФНЧ (рис. 1). Во втором фильтре замкнуты четыре отрезка, обозначенные цифрами I и II, в третьем - шесть (I, II и III), а в четвертом замкнуты все семь узких отрезков. Настройка фильтров осуществлялась подбором ширины проводников у шлейфов и небольшим смещением точки их подключения относительно середины узких проводников так, чтобы максимумы потерь на отражение в полосе пропускания фильтров были близки к $-20 \mathrm{~dB}$. Чтобы не загромождать рисунок, на нем показана частотная зависимость потерь на отражение только для четвертого фильтра.

Для всех четырех конструкций фильтров в табл. 1 приведены значения центральной частоты полосы пропускания $f_{0}$, ее относительной ширины $\Delta f / f_{0}$, измеренной по уровню $-3 \mathrm{~dB}$ от уровня минимальных потерь, которые не превышали $0.4 \mathrm{~dB}$, а также коэффициентов крутизны 

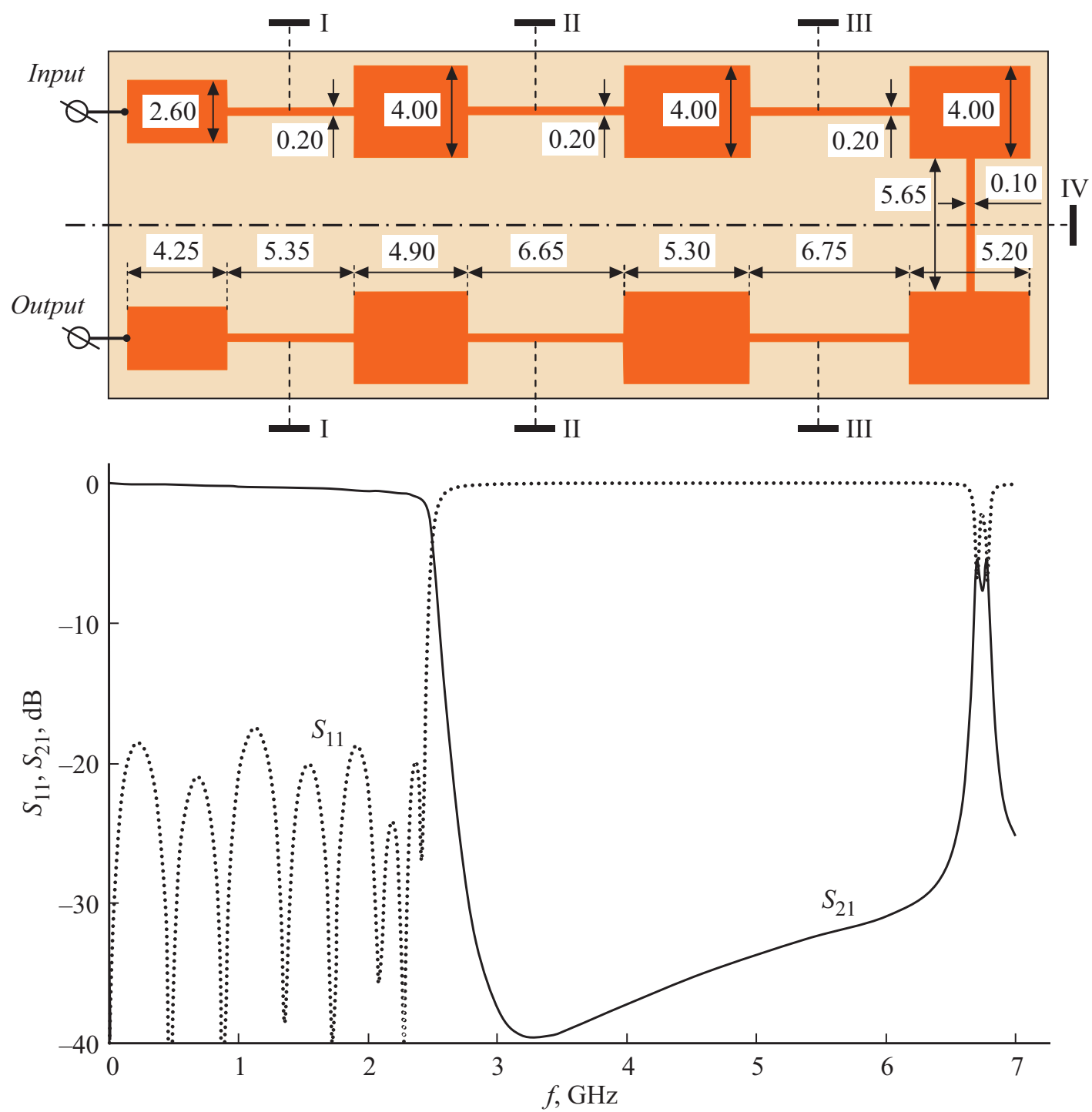

Рис. 1. Топология полоскового проводника фильтра нижних частот (размеры регулярных отрезков полоскового проводника конструкции представлены в $\mathrm{mm}$ ) и частотные зависимости его прямых потерь (сплошная линия) и потерь на отражение (пунктир).

низкочастотного $k_{l}$ и высокочастотного $k_{h}$ склонов АЧХ, вычисленных по формулам [11]:

$$
k_{l}=\frac{\Delta f / 2}{\Delta f_{30}^{l}-\Delta f / 2}, \quad k_{h}=\frac{\Delta f / 2}{\Delta f_{30}^{h}-\Delta f / 2},
$$

где $\Delta f_{30}^{l}$ и $\Delta f_{30}^{h}$ - полосы частот, измеренные от центральной частоты до низкочастотного или высокочастотного склона АЧХ по уровню $-30 \mathrm{~dB}$.

Из таблицы видно, что центральная частота полосы пропускания для первой конструкции фильтра $f_{0}=1.4 \mathrm{GHz}$ и равномерно увеличивается на $100 \mathrm{MHz}$ для каждой последующей конструкции. При этом также равномерно уменьшается относительная ширина полосы пропускания $\Delta f / f_{0}$ (от $150 \%$ для первой конструкции до 90\% для четвертой). Из таблицы также видно, что крутизна высокочастотного склона АЧХ у всех фильтров выше крутизны низкочастотного склона, однако с уменьшением относительной ширины полосы пропускания крутизна склонов постепенно выравнивается и форма АЧХ приближается к симметричной. Важно отметить, что во всех рассмотренных конструкциях изменением длины шлейфов можно плавно регулировать в небольших пределах ширину полосы пропускания устройств. Однако в четвертой конструкции фильтра при одновременном укорачивании длины всех семи замыкающих шлейфов относительную ширину полосы пропускания можно уменьшить в 3 раза от 90 до 30\%.

Экспериментальная проверка работоспособности разработанных сверхширокополосных фильтров проводилась на четвертой из рассмотренных конструкций, в которой все семь шлейфов замкнуты на экран. В качестве подложки экспериментального образца устройства использовалась пластина из поликора толщиной $1 \mathrm{~mm}$. Для определенности были заданы центральная частота полосы пропускания фильтра $f_{0}=2 \mathrm{GHz}$ и ее относи- 


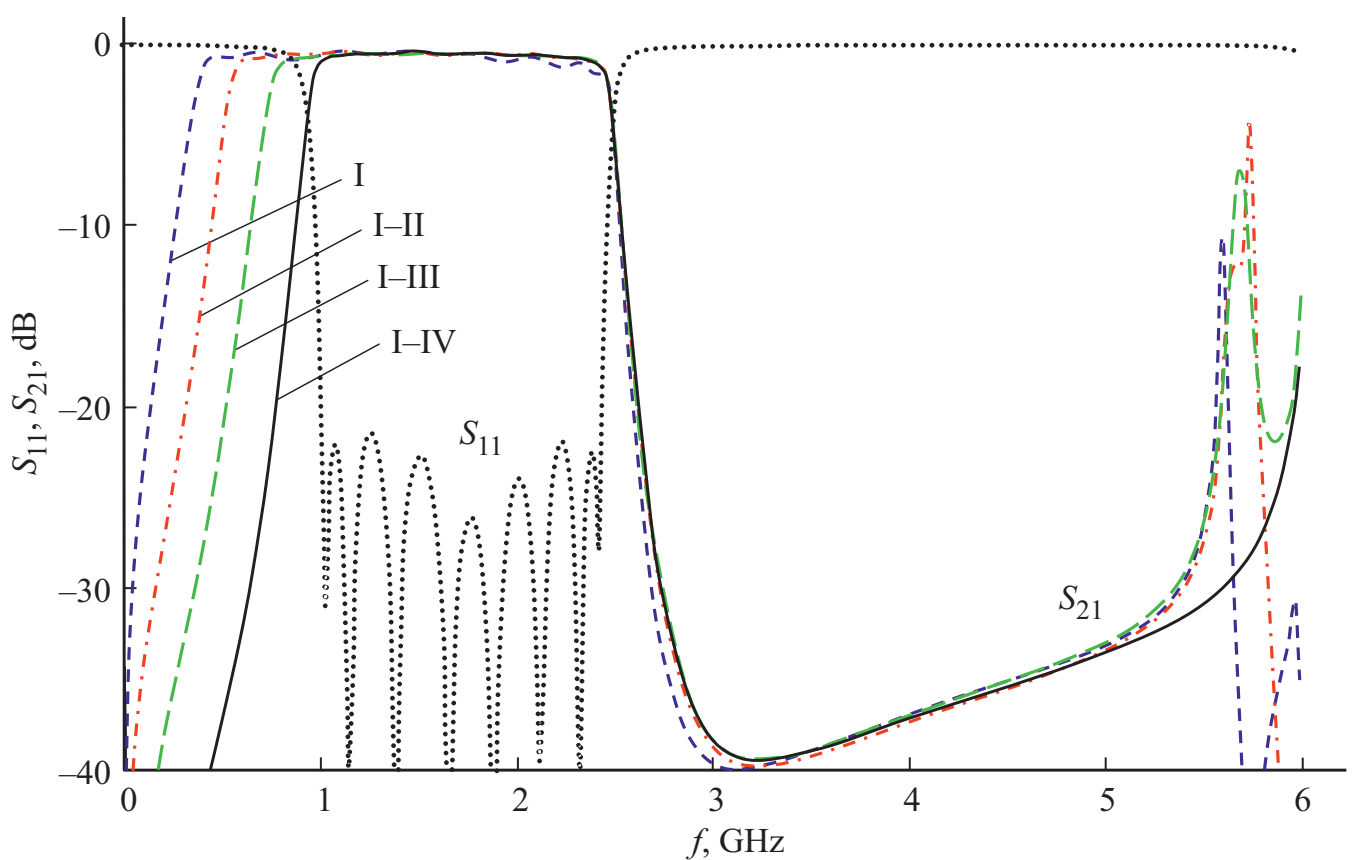

Рис. 2. АЧХ полосно-пропускающих фильтров при замыкании на экран части или всех узких отрезков нерегулярного проводника ФНЧ.

Таблица 1. Параметры амплитудно-частотных характеристик четырех конструкций полосно-пропускающих фильтров

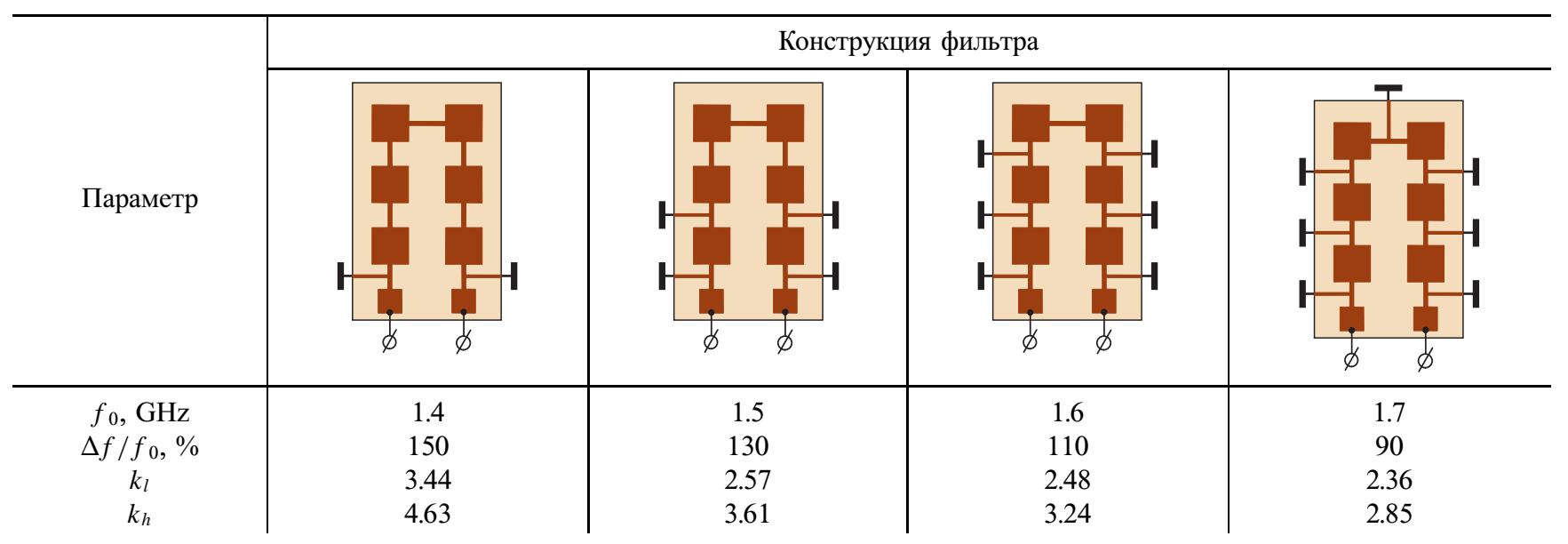

тельная ширина $\Delta f / f_{0}=70 \%$. Параметрический синтез конструкции проводился путем численного электродинамического анализа ее 3D-модели в пакете программ „CST Studio Suite“ так, чтобы все максимумы потерь на отражение в полосе пропускания были на уровне $-20 \mathrm{~dB}$.

Размеры топологии проводников синтезированной конструкции фильтра представлены на рис. 3, $a$ (в миллиметрах), а его рассчитанная частотная зависимость прямых потерь показана сплошной линией 1 на рис. $3, b$. Важно отметить, что на концах шлейфов рассмотренной конструкции присутствуют квадратные площадки размером $1 \times 1 \mathrm{~mm}$ для увеличения прочности проводников при пайке, как и на концах портов, которые для удобства подключения фильтра выведены на противоположные стороны подложки. Отметим также, что в фильтре между широкими отрезками проводников входного и выходного резонаторов добавлен прямоугольный проводник такой же длины, имеющий зазоры с ними по $0.3 \mathrm{~mm}$ (рис. 3,a). Проводник обеспечивает дополнительную связь между этими резонаторами, благодаря которой вблизи высокочастотного склона АЧХ формируется полюс затухания $[9,10]$, значительно увеличивающий крутизну склона, что хорошо видно на рис. $3, b$.

Конструктивные параметры (рис. 3,a), полученные в результате синтеза фильтра по заданным параметрам полосы пропускания, использовались при изготовлении топологии проводников на металлизированной подложке 

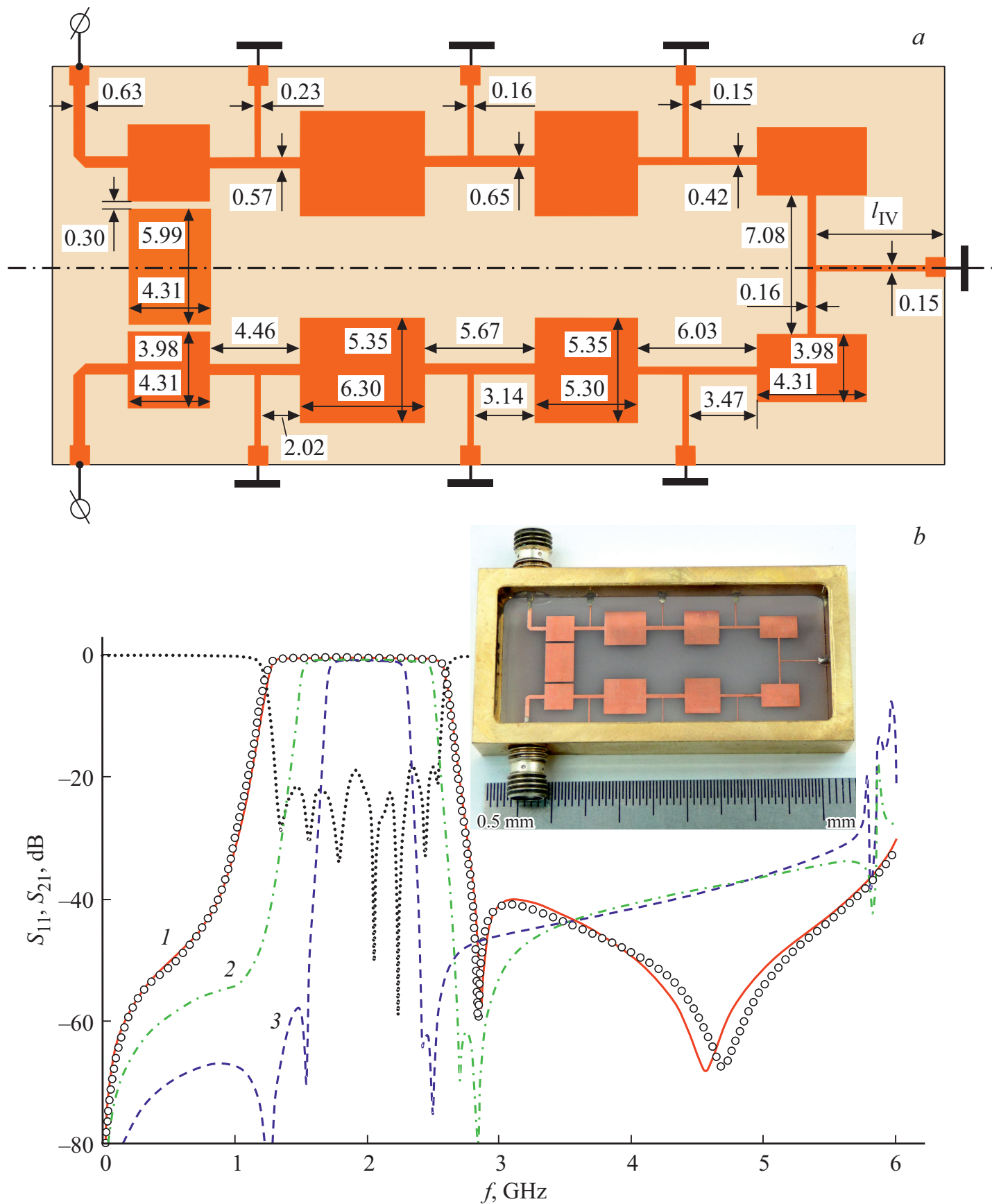

Рис. 3. $a$ ) Топология проводников с размерами (в $\mathrm{mm})$ регулярных участков синтезированного фильтра. $b)$ Линии - частотные зависимости прямых потерь фильтров с $\Delta f / f_{0}=70(1), 50(2)$ и 30\% (3). Точки - измеренные АЧХ экспериментального образца фильтра с $\Delta f / f_{0}=70 \%$. На вставке - его фотография.

из поликора размером $46 \times 21 \mathrm{~mm}$. Полученная микрополосковая структура припаивалась нижним основанием (экраном) ко дну металлического корпуса, а затем контактные площадки шлейфов припаивались к боковым стенкам корпуса. Фотография изготовленного экспериментального образца представлена на вставке к рис. $3, b$. Измеренные на векторном анализаторе цепей R\&S ZVA-40 частотные зависимости прямых потерь $S_{21}$ и потерь на отражение $S_{11}$ показаны на рис. $3, b$ светлыми точками и пунктиром соответственно. Отметим не только достаточно хорошее согласие рассчитанных и измеренных частотных характеристик фильтра, но и одинаковые в теории и эксперименте значения минимальных потерь в полосе пропускания устройства $L_{\min }=0.4 \mathrm{~dB}$.

Как было отмечено выше, одновременное укорачивание длин всех шлейфов в рассматриваемой конструкции приводит к уменьшению ширины ее полосы пропускания. На рис. 3, $b$ представлены АЧХ фильтров, имеющих относительную ширину полосы пропускания 
Таблица 2. Параметры АЧХ фильтров на основе конструкции со всеми замкнутыми на экран шлейфами длиной $l_{\mathrm{I}}-l_{\mathrm{IV}}$, настроенных на центральную частоту полосы пропускания $2 \mathrm{GHz}$

\begin{tabular}{c|c|c|c|c|c}
\hline$l_{\mathrm{I}-\mathrm{III}}, \mathrm{mm}$ & $l_{\mathrm{IV}}, \mathrm{mm}$ & $\Delta f / f_{0}, \%$ & $L_{\mathrm{min}}, \mathrm{dB}$ & $k_{l}$ & $k_{h}$ \\
\hline 4.71 & 6.65 & 70 & 0.4 & 2.85 & 4.00 \\
1.78 & 2.80 & 50 & 0.7 & 3.06 & 4.14 \\
1.06 & 1.70 & 30 & 0.9 & 3.22 & 4.46
\end{tabular}

$50 \%$ (линия 2) и 30\% (линия 3). Заметим, что в конструкциях этих фильтров из-за малой длины шлейфов (табл. 2) потребовалось отрезки узких проводников, которые шлейфами соединяются с экраном (рис. 3,a), сместить от центров к краям широких проводников. При этом расстояние от краев широких проводников до краев подложки всегда превышало $1 \mathrm{~mm}$, т. е. было больше ее толщины. Для объективности сравнения характеристик фильтры настраивались параметрическим синтезом на ту же центральную частоту $f_{0}=2 \mathrm{GHz}$ также в пакете программ „СST Studio Suite“.

В табл. 2 для фильтров, амплитудно-частотные характеристики которых представлены на рис. $3, b$, приведены не только длины шлейфов, но и основные параметры АЧХ. Как и следовало ожидать, уровень минимальных потерь в полосе пропускания $L_{\min }$ с уменьшением ее ширины монотонно растет. Крутизна высокочастотного склона АЧХ $k_{h}$ у всех фильтров существенно больше $k_{l}$ благодаря полюсу затухания, образованному за счет специально созданной связи между крайними резонаторами. Однако с уменышением ширины полосы пропускания фильтра зазоры между широкими проводниками внутренних резонаторов микрополосковой структуры уменьшаются, при этом образуются дополнительные связи между несоседними резонаторами, а в результате на АЧХ фильтров появляются новые полюса, которые увеличивают крутизну низкочастотного склона.

Таким образом, разработаны новые конструкции полосно-пропускающих фильтров на основе микрополоскового ФНЧ, состоящего из чередующихся отрезков широких (низкоомных) и узких (высокоомных) проводников, в котором часть или все высокоомные проводники соединены шлейфами с экраном. Исследования таких конструкций показали возможность реализации на их основе фильтров с относительными ширинами полос пропускания от 30 до 150\%, отличающихся простотой и высокой технологичностью в изготовлении. При этом устройства обладают сравнительно высокими частотноселективными свойствами, что обусловлено легкой возможностью создавать в разработанных конструкциях дополнительные связи между несоседними резонаторами, благодаря которым на частотных зависимостях прямых потерь появляются полюса затухания, значительно увеличивающие крутизну склонов АЧХ. Измеренные характеристики экспериментального образца фильтра с относительной шириной полосы пропускания 70\%, изготовленного на керамической подложке из поликора, показали хорошее согласие с расчетными характеристиками, полученными с помощью электродинамического анализа 3D-модели конструкции. Приведенные выше факты указывают на перспективность использования предложенных конструкций для создания сверхширокополосных фильтров, в которых существует большая потребность, в частности, в высокоскоростных системах радиосвязи.

\section{Финансирование работы}

Работа выполнена при поддержке Министерства науки и высшего образования РФ (государственное задание FEFE-2020-0013).

\section{Конфликт интересов}

Авторы заявляют, что у них нет конфликта интересов.

\section{Список литературы}

[1] C.-L. Hsu, F.-C. Hsu, J.-T. Kuo, in IEEE MTT-S International Microwave Symposium Digest (Long Beach, CA, 2005), p. 679-682. DOI: 10.1109/MWSYM.2005.1516698

[2] Б.А. Беляев, А.М. Сержантов, Ан.А. Лексиков, Я.Ф. Бальва, Е.О. Грушевский, Письма в ЖТФ, 46 (16), 7 (2020). DOI: 10.21883/PJTF.2020.16.49845.18357

[3] Ya.A. Kolmakov, I.B. Vendik, in 35th Eur. Microwave Conf.2005. Conf. Proc. (Paris, 2005), vol. 1, p. 21-24. DOI: 10.1109/EUMC.2005.1608783

[4] S. Shang, B. Wei, B. Cao, X. Guo, X. Wang, L. Jiang, IEEE Trans. Appl. Supercond., 29 (4), 1500105 (2019). DOI: 10.1109/TASC.2018.2880331

[5] Б.А. Беляев, С.А. Ходенков, Ан.А. Лексиков, В.Ф. Шабанов, ДАН, 474 (6), 682 (2017). DOI: $10.7868 / \mathrm{S} 0869565217180062$

[6] R. Zhang, S. Luo, L. Zhu, IEEE Trans. Microwave Theory Tech., 65 (3), 815 (2017). DOI: 10.1109/TMTT.2016.2636825

[7] Y. Zhu, K. Song, Y. Fan, IEEE Access, 7, 117219 (2019). DOI: 10.1109/ACCESS.2019.2928342

[8] K.-D. Xu, D. Li, Y. Liu, IEEE Microwave Wireless Comp. Lett., 29 (2), 107 (2019). DOI: 10.1109/LMWC.2019.2891203

[9] Б.А. Беляев, С.А. Ходенков, В.Ф. Шабанов, ДАН, 485 (1), 27 (2019). DOI: https://doi.org/10.31857/S0869-5652485127-32

[10] B.A. Belyaev, A.M. Serzhantov, Y.F. Bal'va, V.V. Tyurnev, A.A. Leksikov, R.G. Galeev, Microwave Opt. Technol. Lett., 56 (9), 2021 (2014). DOI: 10.1002/mop

[11] Б.А. Беляев, А.А. Лексиков, В.В. Тюрнев, Радиотехника и электроника, 49 (11), 1315 (2004). 